\title{
Correspondence
}

Psychological Medicine, 46 (2016).

doi:10.1017/S0033291716000799

First published online 10 June 2016

\section{Avoiding a lost opportunity for psychological medicine: importance of chimpanzee research to the National Institutes of Health portfolio}

In November 2015, Dr Francis Collins, the director of the National Institutes of Health (NIH), made the unilateral decision to retire all NIH-owned chimpanzees from biomedical research (http://www.nih.gov/about-nih/ who-we-are/nih-director/statements/nih-will-no-longersupport-biomedical-research-chimpanzees). More recently, Dr Collins and the NIH quietly made the decision to limit the types of research with captive chimpanzees that the NIH will fund. Specifically, in the 9 February 2016 Federal Register (http://www.feder alregister.gov/articles/2016/02/09/2016-02554/the-use-ofchimpanzees-in-nih-supported-research), we learned that the NIH will limit even the most minimally noninvasive procedures [e.g. magnetic resonance imaging (MRI) scans or possibly even separating animals temporarily for the administration of cognitive tests]. Unfortunately, these decisions are inconsistent with the Institute of Medicine's (IOM; Altevogt et al. 2011) report on the value and need of chimpanzees to biomedical and behavioral research as well as the NIH's own working group (http:/grants.nih.gov/grants/guide/ notice-files/NOT-OD-12-025.html), both of which highlight the unique value of chimpanzee research to advancing our understanding of human conditions.

Indeed, our research team, as well as others, have demonstrated that chimpanzees are an excellent model species for multilevel behavioral, genetic and neuroscientific research on the pathophysiology of basic transdiagnostic processes (Hopkins et al. 2014; Latzman et al. $2016 a, b)$. These recent decisions will clearly result in lost opportunities for advancing our understanding of the nature, causes, mechanisms and treatments of mental illness, particularly in ways consistent with the recent National Institute of Mental Health (NIMH) Research Domain Criteria (RDoC) Initiative (Insel et al. 2010; Cuthbert \& Insel, 2013). We are thus deeply concerned about the consequences of these decisions to severely limit the ability to utilize this invaluable research resource in the future and believe the scientific community needs to voice its concern.

The RDoC Initiative aims to elucidate the neurobiological basis of mental illness through bootstrapping conceptions of psychopathology from an understanding of the neurobiological basis of transdiagnostic behaviors (Insel et al. 2010; Cuthbert \& Insel, 2013). As described by Krystal (2016), the study of the neurobiology of behaviors naturally expressed by humans and other species (i.e. neuroethology) represents an important research platform for accessing aspects of the biology of complex behaviors consistent with the RDoC Initiative. Indeed, the RDoC Initiative explicitly encourages investigators to utilize animal models to investigate various constructs within the various specified domains. In our view, chimpanzees represent an unparalleled animal model for such investigations.

Recent work by our research team exemplifies the promise held by research with chimpanzees that falls well within IOM guidelines and follows the goals and aims of the RDoC Initiative. For example, we have found a largely similar, heritable personality structure in chimpanzees that resembles that found in humans (Hopkins et al. 2012; Latzman et al. 2014, 2015a). Variation in chimpanzees' personality also has parallel neuroanatomical and genetic correlates as those reported in humans (Latzman et al. 2015b); these findings serve to support the cross-species nature of trait personality and the potential mechanisms that underlie their expression. Further, using diffusion tensor imaging (DTI), we (Latzman et al. 2015c) recently examined the relationship between white matter connectivity and delay of gratification (DG) abilities, processes known to prospectively predict a host of problematic outcomes including, potentially most notably, attention-deficit/hyperactivity disorder and substance use disorders. Higher white matter connectivity between the caudate and right dorsal prefrontal cortex (PFC) was associated with individual variation in DG, suggesting the integrity of white matter connectivity between striatal and PFC regions to be critical for inhibitory control. This research has clear translational value to humans with perturbations on this circuit, likely bearing transdiagnostic implications for a variety of behavioral problems characterized by weak inhibitory control.

Lastly, for some phenotypes, chimpanzees are essentially the only alternative model species for comparative studies with humans. For instance, chimpanzees (and perhaps other great apes) have been documented to referentially point, intentionally communicate, and initiate and engage in joint attention during intra- and interspecies communication (Leavens, 2012). Similarly, chimpanzees, but not more distantly related primates, show mirror self-recognition (Anderson \& Gallup, 
2011), perspective-taking abilities and some basic forms of empathy (De Waal, 2008). All of these traits are critically important behaviors that distinguish typically developing children from with or at risk for a diagnosis of autism or related neurodevelopmental disorders (Mundy, 2016). More generally, within the Social Process Domain of the RDoC, behaviors such as joint attention, perceptions of self and perception of others are listed as key phenotypes. We are now facing the prospect that continued studies in these important domains of research will be significantly constrained or outright eliminated by the NIH for no sound scientific or ethical reason.

What is most disheartening is that the vast majority of behavioral, genomic and neuroscience research we and others have performed falls well within the ethical framework of scientifically justifiable research with chimpanzees outlined by the IOM as well as the NIH Working Group. Indeed, some of this work was singled out in the IOM report as being highly translational and of the highest ethical standards. Indeed, for procedures such as MRI and positron emission tomography (PET) imaging, the chimpanzees are trained to voluntarily present for a shot for the anesthesia. The scans are collected at the same time the individuals are immobilized for their annual veterinary examination, something our group implemented and followed long before the IOM recommended this as a standard procedure. Likewise, according to the IOM and NIH Working Group, administering behavioral or cognitive tests to captive chimpanzees is inherently enriching, yet the NIH is proposing to eliminate these procedures and insisting that only observational methods be used with chimpanzees.

In summary, if the recent NIH decisions and recommendations remain unchanged, this will clearly result in lost opportunities for advancing our understanding of the pathophysiology of psychopathology in humans but also have a negative impact on the well-being of captive chimpanzees. Indeed, funds have already been spent to establish the chimpanzee genome (Chimpanzee Sequencing and Analysis Consortium, 2005) and the National Chimpanzee Brain Resource (NCBR), important scientific resources in psychological medicine. Further, the NIH owns and currently supports more than 350 chimpanzees currently residing in research and sanctuary settings in the USA. It is quite concerning that the NIH continues to financially support these apes and yet is putting in place significant limitations to their use in the types of non-invasive research described here, particularly when the scientific advancements and benefits that might come from these efforts are of significant translational value. The absence of our ability to capture important phenotypes with which to connect genetic and neuroanatomical data would be a tragedy particularly in light of the financial resources already committed and will be used to maintain the captive chimpanzee population for the next 25-30 years. We have an opportunity, and indeed an obligation, to continue to study chimpanzees for the purposes of benefitting both human and chimpanzee physical and psychological health. This goal can be accomplished without the use of invasive methods and by adhering to the highest ethical standards in research with primates. All told, the larger scientific community, and particularly those committed to elucidating basic processes associated with psychiatric health in humans, should embrace the opportunity we currently have and take a stand to not allow this amazing resource to be squandered.

\section{Acknowledgements}

None.

\section{Declaration of Interest}

None.

\section{References}

Altevogt BM, Pankevich DE, Shelton-Davenport MK, Kahn JP (2011). Chimpanzees in Biomedical and Behavioral Research: Assessing the Necessity. The National Academies Press: Washington, DC.

Anderson JR, Gallup Jr GG (2011). Which primates recognize themselves in mirrors? PLoS Biology 9, e1001024.

Chimpanzee Sequencing and Analysis Consortium (2005). Initial sequence of the chimpanzee genome and comparison with the human genome. Nature 437, 69-87.

Cuthbert B, Insel TR (2013). Toward the future of psychiatric diagnosis: the seven pillars of RDoC. BMC Medicine 11, 126.

De Waal FBM (2008). Putting the altruism back into altruism: the evolution of empathy. Annual Review of Psychology 59, 279-300.

Hopkins WD, Donaldson ZR, Young LY (2012). A polymorphic indel containing the RS3 microsatellite in the $5^{\prime}$ flanking region of the vasopressin V1a receptor gene is associated with chimpanzee (Pan troglodytes) personality. Genes, Brain and Behavior 11, 552-558.

Hopkins WD, Keebaugh AC, Reamer LA, Schaeffer J, Schapiro SJ, Young LJ (2014). Genetic influences on receptive joint attention in chimpanzees (Pan troglodytes). Scientific Reports 4, 3774.

Insel TR, Cuthbert B, Garvey M, Heinssen R, Pine DS, Quinn K, Sanislow C, Wang P (2010). Research domain criteria (RDoC): toward a new classification framework for research on mental disorders. American Journal of Psychiatry 167, 748-751.

Krystal JH (2016). Neuroethology as a translational strategy in the era of the NIMH Research Domain Criteria. Psychophysiology 53, 364-366.

Latzman RD, Drislane L, Hecht LK, Brislin S, Patrick CJ, Lilienfeld SO, Freeman HJ, Schapiro SJ, Hopkins WD 
(2016a). A chimpanzee (Pan troglodytes) model of triarchic psychopathy constructs: development and initial validation. Clinical Psychological Science 4, 50-66.

Latzman RD, Freeman HD, Schapiro SJ, Hopkins WD (2015a). The contribution of genetics and early rearing experiences to hierarchical personality dimensions in chimpanzees (Pan troglodytes). Journal of Personality and Social Psychology 109, 889-900.

Latzman RD, Hecht LK, Freeman HD, Schapiro SJ, Hopkins WD (2015b). Investigating the neuroanatomical correlates of personality in chimpanzees (Pan troglodytes): associations between personality and frontal cortex. NeuroImage 123, 63-71.

Latzman RD, Hopkins WD, Keebaugh AC, Young LJ (2014). Personality in chimpanzees (Pan troglodytes): exploring the hierarchical structure and associations with the vasopressin V1A receptor gene. PLoS ONE 9, e95741.

Latzman RD, Taglialatela JP, Hopkins WD (2015c). Delay of gratification is associated with white matter connectivity in the dorsal prefrontal cortex: a diffusion tensor imaging (DTI) study in chimpanzees (Pan troglodytes). Proceedings of the Royal Society: of London: Biological Sciences B 22, 1809-1814.
Latzman RD, Young LJ, Hopkins WD (2016b). Displacement behaviors in chimpanzees (Pan troglodytes): a neurogenomics investigation of the $\mathrm{RDoC}$ negative valence systems domain. Psychophysiology 53, 355-363.

Leavens DA (2012). Joint attention: twelve myths. In Joint Attention: New Developments in Psychology, Philosophy of Mind and Social Neuroscience (ed. A. Seemann), pp. 43-72. MIT Press: Cambridge, MA.

Mundy PC (2016). Autism and Joint Attention: Development, Neuroscience and Clinical Fundamentals. The Guilford Press: New York.

R. D. LATZMAN ${ }^{1}$ AND W. D. HOPKINS ${ }^{2}$

${ }^{1}$ Department of Psychology, Georgia State University, Atlanta, GA, USA

${ }^{2}$ Neuroscience Institute, Georgia State University, Atlanta, GA, USA

Author for correspondence: R. D. Latzman, Department of Psychology, Georgia State University, PO Box 5010, Atlanta, GA 30302-5010, USA.

(Email: rlatzman@gsu.edu) 